\title{
Correlación entre el nivel de dependencia funcional del adulto mayor y nivel de sobrecarga del cuidador primario en un hospital público de Aguascalientes
}

Martínez-García Itzeen Itzamatul Guadalupe, Gallardo-García David, González-Benítez María Fernanda, Guel-Cruz Karla del Rocío, Aguilera-Ibarra Karen Dinhora, González-Flores Silvia Patricia

\section{Resumen}

- Introducción. Las personas dependientes requieren de cuida-

- dos continuos y en ocasiones extenuantes que pueden tener

- repercusiones de salud en su cuidador primario. Objetivo:

- Determinar la correlación del nivel de sobrecarga del cui-

- dador primario en relación con el nivel de dependencia del

- adulto mayor. Metodología: Estudio descriptivo, correlacional,

- transversal y prospectivo, instrumentos utilizados test de Za-

- rit con confiabilidad de 0.84 en Chrombach y la escala de

- Katz con una confiabilidad de 0.98 en alfa de Chrombach,

- para el análisis de datos se utilizó el paquete estadístico

- SPSS 23 ®, usando la prueba de correlación estadística de

- Spearman. Resultados: Muestra de 70 adultos mayores hos-

- pitalizados en un hospital de segundo nivel en el estado de

- Aguascalientes; edad predominante entre 60 y 69 años, pre-

- dominando el sexo masculino; se obtuvo que a mayor nivel

- de dependencia del adulto mayor no existe un aumento del

- nivel de sobrecarga del cuidador primario, mediante un coe-

- ficiente de correlación rho de Spearman de $0.070 p<0.05$.

- Conclusión: La dependencia funcional del adulto mayor no

- significa un incremento en el nivel de sobrecarga del adulto

- mayor, sino que se tiene que valorar su perfil patológico, y

- tiempo de evolución de las enfermedades. LUXMÉDICA, AÑO

- 12, NÚMERO 35, ENERO-ABRIL 2017, PP 3-10

Palabras claves: Adulto mayor, Dependencia, Sobrecarga.

\section{Abstract}

Introduction: Dependant people require continuous and sometimes strenuous care, which can have health repercussions on their primary caregiver. Objective: To determine the correlation of the primary caregiver's level of overload in relation with the level of dependence of the elderly. Methods: Descriptive, correlational, cross-sectional and prospective study; Zarit test with reliability of 0.84 in Chrombach and Katz scale with a reliability of 0.98 in Chrombach 's alpha were used; statistical package SPSS 23 @ was used for data analysis, Spearman's statistical correlation test was used. Results: Sample of 70 elders hospitalized in a second level hospital in the state of Aguascalientes, predominant age between 60 and 69 years, mainly male; a greater level of dependence of the elderly does not increase the level of the overload of the primary caregiver, using a Spearman rho correlation coefficient of $0.070 p<0.05$. Conclusion: The functional dependence of the elderly does not mean an increase in the level of the overload of the primary caregiver; therefore the profile and evolution of disease must be assessed. LUXMÉDICA, AÑO 12, NÚMERO 35, ENERO-ABRIL 2017, PP 3-10

* Pasantes de Enfermería de Servicio Social del Centro de Ciencias de la Salud de la Universidad Autónoma de Aguascalientes.

** Maestra en Ciencias Biomédicas de Enfermería y Profesora Investigadora y del Departamento de Enfermería del Centro de Ciencias de la Salud de la Universidad Autónoma de Aguascalientes.

Fecha de recibido: 20 de julio 2016

Fecha de aceptación: 6 de noviembre 2017

Correspondencia: MCE Silvia Patricia González Flores. Departamento de Enfermería. Centro de Ciencias de la Salud. Universidad Autónoma de Aguascalientes. Avenida Universidad No. 940. Edificio 105. Ciudad Universitaria. Código postal 20131. Aguascalientes, Ags., México Teléfono 9108437, correo electrónico: spgonza@correo.uaa.mx 


\section{Introducción}

El aumento de esperanza de vida al nacer provoca que la dinámica demográfica en México a futuro presente cambios, en la que se ve involucrado un aumento en el número de adultos mayores y con ello el número de personas dependientes funcionalmente y demográficamente. ${ }^{1}$ Los científicos sociales especializados en el estudio del envejecimiento describen, cronológicamente y funcionalmente, tres grupos de adultos mayores: "viejo joven" de 60 y 74 años, siendo por lo general activos, "viejo viejo" de 75 a 84 años; ambos grupos constituyen la tercera edad y "viejo de edad avanzada" de 85 años en adelante, comprende la cuarta edad; es muy probable que se sientan débiles, presenten enfermedades discapacitantes que le dificulten la realización de las actividades de la vida diaria. ${ }^{2}$

En Chile el Servicio Nacional del Adulto Mayor (SENAMA), estipula que las definiciones de dependencia se enmarcan "dentro de las limitaciones o incapacidad para efectuar las actividades de la vida diaria", y Barthel la describe como "la incapacidad funcional en relación con las actividades de la vida diaria". Se considera una situación específica de discapacidad, observándose una limitación severa de la persona para realizar una determinada actividad y la necesidad de recibir ayuda personal o técnica para poder interactuar con el medio ambiente, por lo que la relación discapacidad y dependencia constituye una situación compleja de salud. ${ }^{3}$

Las personas dependientes requieren de cuidados continuos y en ocasiones extenuantes para las personas que se encargan de atenderlos; en relación a lo anterior (Astudillo, 2008) se ha definido al cuidador primario, como la persona que atiende en primera instancia las necesidades físicas y emocionales de un enfermo, papel que por lo general lo juegan el/ la esposo/a, hijo/a, un familiar cercano o alguien que es significativo para el paciente, además plantea que el cuidador primario asume la responsabilidad total del paciente ayudándole a realizar todas las actividades que éste por sí mismo no puede llevar a cabo; por lo general no recibe ayuda económica ni capacitación previa para la atención del enfermo. ${ }^{4}$

Un estudio reciente reportó que existe una relación entre el grado de la sobrecarga del cuidador con el grado de dependencia, la cual está estrechamente relacionada con el tiempo de ejercer esta función, las patologías con las que cuenta el cuidador y con el grado de dependencia de los pacientes cuidados. ${ }^{5}$ En otro estudio encontraron que a menor grado de dependencia del adulto mayor, menor es el nivel de sobrecarga subjetiva del agente de cuidado dependiente. ${ }^{6}$

Bello, en su tesis llamada Sobrecarga del cuidador primario de pacientes con enfermedades crónico degenerativas, identifica el grado de sobrecarga que presentan los cuidadores primarios de pacientes con enfermedades crónico-degenerativas, donde con- 
cluye que, según la teoría socio cognitiva, la funcionalidad del adulto mayor es un resultado de la interacción de la salud del adulto mayor con su entorno familiar e institucional. ${ }^{5}$

El objetivo de este estudio fue determinar la correlación del nivel de sobrecarga del cuidador primario con el nivel de dependencia del adulto mayor.

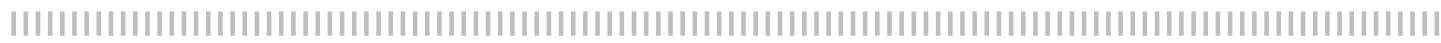

\section{Material y métodos}

Estudio descriptivo, correlacional, transversal, prospectivo, realizado en un hospital público en la ciudad de Aguascalientes en pacientes de los servicios de medicina interna (hombres y mujeres) y de cirugía. Se incluyeron todos los adultos mayores de 60 años con dependencia funcional y a sus cuidadores primarios, excluyendo a aquellos adultos mayores sin dependencia funcional. Se obtuvo una muestra de 70 adultos mayores dependientes y 70 cuidadores primarios por muestreo por conveniencia.
Los instrumentos utilizados fueron el índice de Katz ${ }^{7}$ para la valoración de las actividades de la vida diaria que cuenta con seis ítems con una confiabilidad de 0.98 en alfa de Chrombach y la escala de sobrecarga de Zarit \& Zarit, $^{8}$ de 22 ítems con 0.84 en Chrombach. El análisis de datos se realizó por medio del paquete estadístico SPSS RM v23. Los resultados se reportan con medidas de tendencia central, porcentajes, frecuencias, mínimos, máximos, y la prueba de Spearman.

\section{| | | | | | | | | | | | | | | | | | | | | | | | | | | | | | | | | | | | | | | | | | | | | | | | | | | | | | | | | | | | | | | | | | | | | | | | | | | | | | | | | | | | | | | | | | | | | | | | | | | | | | | | | | | | | | | | | ||}

\section{Resultados}

La población de estudio fue de 70 adultos mayores dependientes y 70 cuidadores primarios. Predominó en un 54\% el adulto de sexo masculino con un rango de edad entre 60-69 años. El 58\% de los sujetos de estudio no cuentan con estudios escolares; la causa de ingreso a la unidad hospitalaria fue debida a traumas y fracturas con el $17 \%$.

En cuanto a cuidador primario predominó el sexo femenino con el $80 \%$ con un rango de edad entre 31 y 60 años. El $64 \%$ de los cuidadores primarios se encuentran desempleados, el $50 \%$ de ellos tienen el rol de hijo; el $43 \%$ del total de los cuidadores primarios, aparte de cuidar a su paciente, cuidan a otra persona; y sólo el $1 \%$ de los cuidadores perciben una remuneración económica. El adulto mayor hospitalizado con dependencia mostró una tendencia a tener dependencias más severas, tal así que $46 \%$ de la muestra reflejó un nivel de dependencia más severa: la invalidez (figura1). 


\section{Figura I}

Nivel de dependencia del adulto mayor dependiente, en un hospital general de Aguascalientes

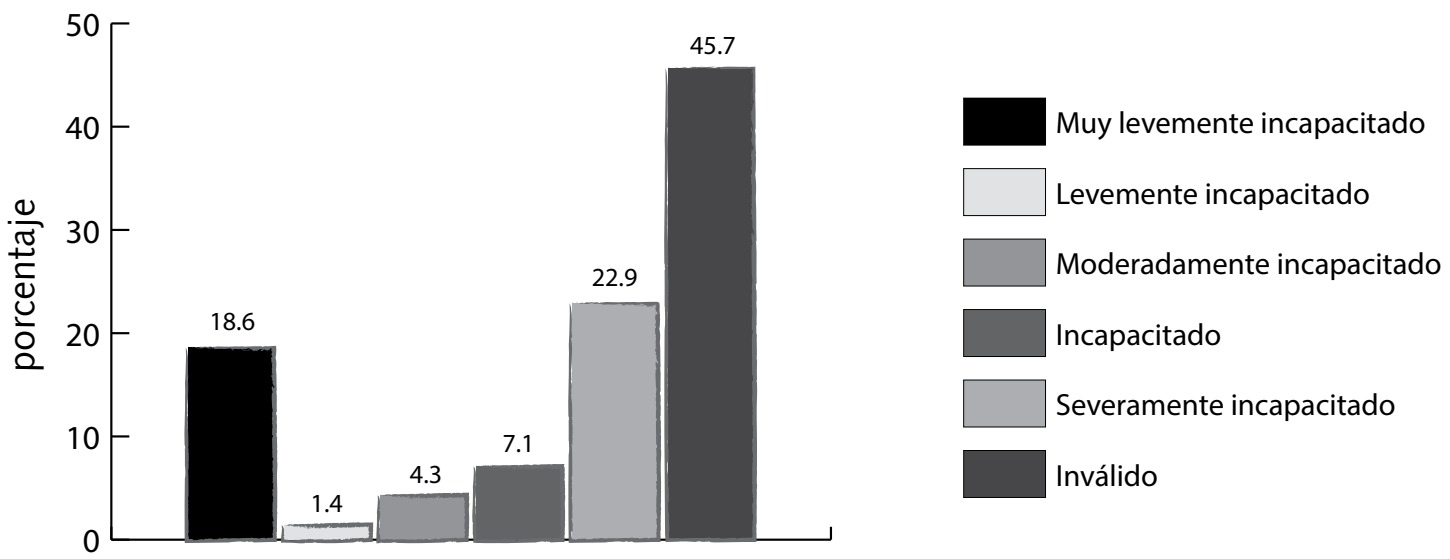

Fuente: Nivel de dependencia en porcentaje del adulto mayor dependiente, en un hospital general de Aguascalientes febrero-marzo 2016. $n=70$.

Con el instrumento utilizado (Zarit) para dencias funcionales severas, como lo son la demostrar sobrecarga del cuidador prima- invalidez y severamente incapacitado, en rio, esta arrojó que el $52.86 \%$ no posee los adultos mayores (figura 2). sobrecarga pese a la tendencia de depen-

\section{Figura 2}

Nivel de sobrecarga del cuidador primario del adulto mayor dependiente, en un hospital general de Aguascalientes

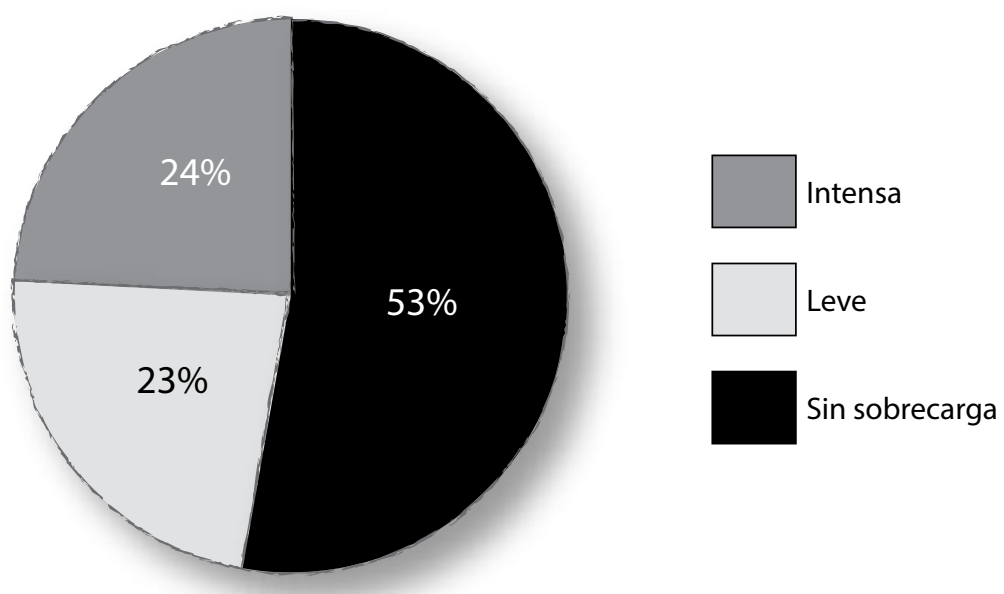

Fuente: Nivel de sobrecarga del cuidador primario del adulto mayor dependiente, en un hospital general en Aguascalientes febrero-marzo 2016. $n=70$. 
En comparación sobre los servicios hospitalarios donde se realizó la investigación, se observaron porcentajes similares entre Medicina Interna Hombres y Cirugía, mientras que en Medicina Interna Mujeres predomina no sobrecarga del cuidador primario; hecho que puede verse reflejado de acuerdo con la diferencia de tiempo dedicado al cuidado del adulto mayor (figura 3).

\section{Figura 3}

Nivel de sobrecarga del cuidador primario del adulto mayor dependiente por servicio en un hospital general de Aguascalientes

Medicina interna mujeres $\square$ Medicina interna hombres $\square$ Cirugía

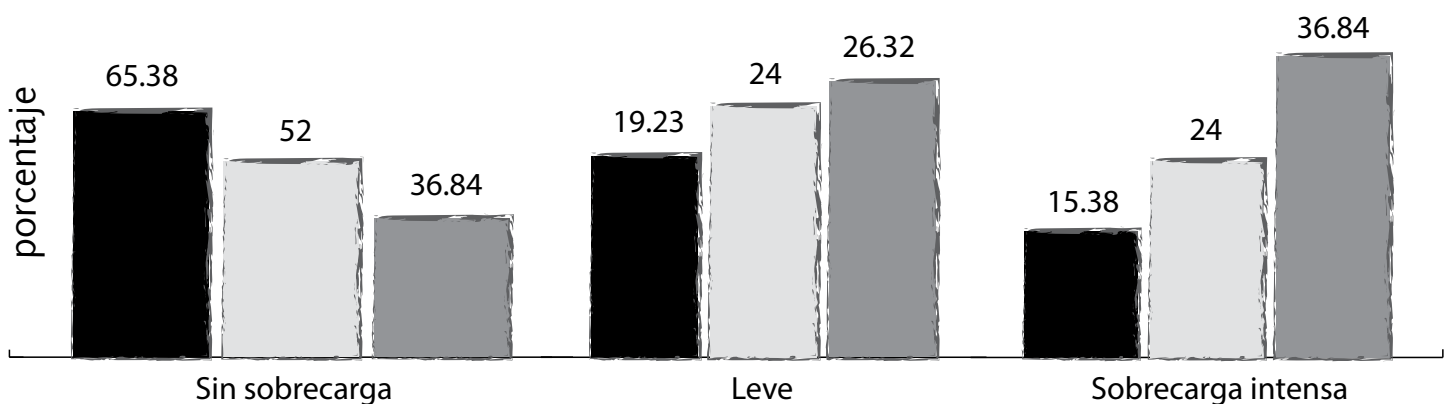

Fuente: Nivel de sobrecarga del cuidador primario del adulto mayor dependiente por servicio (\%) en un Hospital General en Aguascalientes febrero-marzo 2016. $n=70$.

Referente al propósito del estudio se estableció que a mayor nivel de dependencia del adulto mayor no existe un aumento del nivel de sobrecarga del cuidador primario, por lo que en este caso fue certera la hipótesis nula, con un coeficiente de correlación de Spearman de .070 con intervalo de confianza del 95\% $(p=0.5)$ (tabla 1).

\section{Tabla I}

Coeficiente de correlación de Spearman con las variables de nivel de dependencia funcional del adulto mayor y el nivel de sobrecarga del cuidador primario en un hospital público.

\begin{tabular}{|c|c|c|c|}
\hline Spearman's rho & & NDAD & NSCP \\
\hline \multirow[t]{6}{*}{ Nivel de dependencia del adulto mayor (NDAD). } & Coeficiente de correlación & 1.000 & .070 \\
\hline & Significancia. (2-tailed) & . & .564 \\
\hline & Total & 70 & 70 \\
\hline & Error estándar & .000 & .115 \\
\hline & Inferior & 1.000 & -.159 \\
\hline & Intervalo de confianza & 1.000 & .283 \\
\hline \multirow[t]{6}{*}{ Nivel de sobrecarga del cuidador primario (NSCP). } & Coeficiente de correlación & .070 & 1.000 \\
\hline & Significancia (2-tailed) & .56 & \\
\hline & Total & 70 & 70 \\
\hline & Error estándar & .115 & .000 \\
\hline & Inferior & -.159 & 1.000 \\
\hline & Intervalo de confianza & .283 & 1.000 \\
\hline
\end{tabular}

Fuente: Correlación entre el nivel de dependencia funcional del adulto mayor y nivel de sobrecarga del cuidador primario en un hospital público de Aguascalientes 


\section{Discusión}

De acuerdo con los datos y resultados referente a características sociodemográficas del adulto mayor dependiente, se difiere con Martín en su estudio en donde muestra que el $84 \%$ fueron del género femenino; en relación a la edad, se coincide, pues muestra un mayor porcentaje de adultos mayores de 60 años; en cuanto a las características sociodemográficas del cuidador primario, se coincide con Carrillo, ${ }^{10}$ el cual refleja que un $88.7 \%$ de los cuidadores primarios son mujeres. Además, se coincide con Flores, ${ }^{3}$ pues $91 \%$ de los cuidadores son mujeres, en el que $49.3 \%$ de los cuidadores son hijos(as) del adulto mayor, mientras que el $23.9 \%$ son cónyuges y se difiere en su estudio en cuanto al nivel de sobrecarga, pues en éste se muestra un porcentaje equitativo de $29.9 \%$ entre los grupos de edad de 46 a 55 años y mayores de 65 años. Con Dávila ${ }^{11}$ se coincide pues encuentra que el $58 \%$ de los cuidadores oscilan entre los 41 y 60 años, en el que el $73.0 \%$ son hijos y sólo el $13.0 \%$ son parejas de los adultos mayores y $28 \%$ de los cuidadores cuenta con empleo; se coincide con Cardona ${ }^{12}$ que demuestra que $73.7 \%$ presentan un rol como amas de casa, y sólo el $5.3 \%$ se encuentran empleados pero se difiere en que el $38.3 \%$ son hijos y $38.7 \%$ son cónyuges. También los resultados van en sintonía con lo que reporta Bello, ${ }^{5}$ pues muestra que el $94 \%$ de los cuidadores no percibe remuneración económica por realizar la actividad del cuidador; de igual manera sucede con Carrillo, ${ }^{10}$ pues reporta que el $73.3 \%$ de los cuidadores tiene una ocupación en el hogar, mientras que sólo $6.6 \%$ cuentan con empleo y, a su vez, también en su estudio, encuentra que el $50.9 \%$ se dedica al cuidado de 24 horas al día. Sucede lo mismo con Morales ${ }^{13}$ pues, según su estudio, el $81.6 \%$ de los cuidadores dedica siete días por semana y $72.4 \%$ de los cuidadores dedica más de 12 horas al cuidado. Al igual que Palomé14 que refle- ja que $54.2 \%$ dedica más de 15 horas de cuidado al adulto, lo cual se traduce en un $89.6 \%$ con respecto al cuidado entre 4 a 7 días a la semana.

En cuanto a los resultados de nivel de dependencia del adulto mayor observamos una predominante concentración entre inválido a severamente incapacitado con un $69 \%$, por lo que se coincide con Bello una vez más, pues encuentra que existe una tendencia del $88 \%$ a dependencias severas. Continuando con el análisis de acuerdo al índice de Katz, ${ }^{7}$ los resultados arrojaron menor dependencia en la variable "comer" con $50 \%$ en comparación del resto de las variables que son de aproximadamente $80 \%$, lo cual afecta los requisitos universales de autocuidado de Orem y coincidiendo con García. ${ }^{15}$ Por otra parte se obtuvo que existe relación entre el grado de dependencia y el aumento de la edad con una $p=0.05$, coincidiendo así con Barrón ${ }^{4}$ y Bello ${ }^{5}$, pues reporta que el aumento de discapacidad, en este caso, dependencia física, aumenta con la edad a consecuencia de una lesión, de una enfermedad o a raíz del mismo proceso de envejecimiento del adulto mayor; esto provoca, en algunas ocasiones, que otro miembro de la familia deba proveerlo de ayuda material y humana, por lo que adquiere el papel de cuidador.

En el nivel de sobrecarga del cuidador primario se obtuvo que $47 \%$ de los cuidadores presentó algún nivel de sobrecarga y $68 \%$ de los adultos mayores tenía un nivel de dependencia elevado o inválido, por lo que se coincide con los resultados que presentaron los cuidadores primarios de pacientes con enfermedades crónicodegenerativas de una UMF en el estudio de Bello, pues el $52 \%$ de los cuidadores presentó algún grado de sobrecarga, de acuerdo a la escala de Zarit, y $88 \%$ de los pacientes cuidados presentó algún grado de dependencia moderada severa. 
Se encontró una tendencia a no tener sobrecarga del cuidador primario, donde $53 \%$ no presentó, $24 \%$ indicó que era leve y $23 \%$ manifestó una sobrecarga intensa, datos que difieren con Ruíz, ${ }^{16}$ el cual reporta que el $61.8 \%$ de los cuidadores presentó cansancio intenso, $19.5 \%$ cansancio leve, y sólo $18.7 \%$ no reportó sobrecarga, resultados posiblemente atribuibles a que ellos utilizaron la variable de tiempo de cuidado en su investigación.

Con respecto a la correlación y la prueba utilizada, se encontró un coeficiente de Spearman de $.070 p<0.05$, lo que supone que no hay ningún tipo de correlación significativa por lo que se coincide con Rodríguez ${ }^{6}$, con una correlación estadística de Spearman de $-0.46 p<0.05{ }^{6}$ se difiere con Bello ${ }^{5}$ quién encontró que a mayor dependencia del paciente, mayor sobrecarga presentan los cuidadores, donde tras una prueba de Chi cuadrada obtuvo un valor de 89 con $p=0.00$; aunque es posible que exista alguna diferencia con este último autor por el uso de la prueba de Bartell en vez de Katz para valorar la dependencia funcional del adulto mayor.

\section{Conclusiones}

Aunque las personas dependientes requieren de cuidados y, en ocasiones, extenuantes, que pueden afectar a los cuidadores primarios. Esto podría ser resultado del hecho de que ellos realizan las actividades que las personas dependientes ya no pueden realizar. Lo anterior no significa que existe un incremento en el nivel de sobrecarga del cuidador primario, sino que existen factores que varían de acuerdo con el perfil patológico, el tiempo de evolución de las enfermedades, el tiempo de cuidado que dedica el cuidador primario, así como si existe más de un cuidador primario. Se encontró un porcentaje, aunque no representativo, de cuidadores primarios con nivel de sobrecarga que van de leve a intensa, por lo que se recomiendan futuras investigaciones a los factores que intervienen a la sobrecarga del cuidador primario.

\section{Bibliografía}

1. INEGI; Los adultos mayores en México: Perfil sociodemográfico al inicio del siglo XXI; Aguascalientes, México; 2005; Citado Enero 2016 http://www.inegi.gob.mx/prod_serv/contenidos/espanol/bvinegi/ productos/ce nsos/poblacion/adultosmayores/Adultos mayores_web2.pdf.

2. Fernández Ālba R., Manrique-Abril F. G.. Rol de la enfermería en el apoyo social del adulto mayor. Enferm. glob. [Internet]. 2010 Jun [citado 2016 Mayo 29]; (19). Disponible en: http://scielo.isciii. es/scielo.php?script =sci_arttext $\&$ pid $=$ S169561412010000200016\&lng=es.

3. Flores E; Nivel de sobrecarga en el desempeño del rol del cuidador familiar de adulto mayor con dependencia severa; RevScielo; Chile; 2012; Citado Enero 2016 Disponible en: http://www.scielo.cl/scielo.php?script $=$ sci_arttext\&pid $=$ S071795532012000100004.
4. Barrón R. Brenda; Desgaste físico y emocional del cuidador primario en Cáncer; Instituto Nacional de Cancerología de México; México, D.F; 2009; Citado: Enero 2016. http://www.incan.org.mx/revistaincan/elementos/documentosPortada/12575 41295.pdf.

5. Bello Pinedo J; Sobrecarga del cuidador primario de pacientes con enfermedades crónico degenerativas; Veracruz, México; 2014; Citado Enero 2016 http:// www.uv.mx/blogs/favem2014/files/2014/06/Tesis- gabriela.pdf.

6. Rodríguez Medina; Sobrecarga del agente de cuidado dependiente y su relación con la dependencia funcional del adulto mayor; UNAM; México, D.F. 2011; Citado 2016.

7. Pérez Reyes $M$; Capacidad funcional del adulto mayor en una institución de primer nivel de atención; Universidad Veracruzana; Veracruz, México 2010; Citado Enero 2016 Disponible en: http://cdigital. uv.mx/bitstream/123456789/29281/1/tesis2.pdf 
8. Montero Pardo X. et al; Escala de carga del cuidador de Zarit: Evidencia de validez en México: Rev. Psicoonclología Vol. 1; México; 2011; Citado enero 2016 Disponible en: https://revistas.ucm.es/index. php/PSIC/article/viewFile/44918/42834.

9. Martín Silva Evelin; Percepción de la funcionalidad del adulto mayor a través de la teoría socio cognitiva de Albert Bandura en la Unidad de Medicina Familiar Número 1 del Instituto Mexicano del Seguro Social, Delegación Aguascalientes; Universidad Autónoma de Aguascalientes 2015; Citado: Mayo 2016. Disponible en: http://bdigital.dgse.uaa.mx:8080/xmlui/ handle/123456789/325

10. Carrillo González Gloria Mabel, Sánchez Herrera Beatriz, Barrera Ortiz Lucy. Habilidad de cuidado de cuidadores familiares de niños y adultos conenfermedad crónica. IndexEnferm [Internet]. 2014 Sep; Citado 2016 Mayo 24] ? 23(3): 129133 Disponible en: http://scielo.isciii.es/scielo. php?script $=$ sci_arttext $\&$ pid $=$ S1132129620140002 $00003 \&$ lng $=$ es.http://dx.doi.org/10.4321/S113212962014000200003

11. Dávila Mendoza R, Rivera Mora H, González Pedraza Avilés A, Calidad de vida de los cuidadores primarios de pacientes geriátricos de la Clínica de Medicina Familiar Dr. Ignacio Chávez, ISSSTE. Revista de Especialidades Médico-Quirúrgicas 20111627-32. Disponible en: http://www.redalyc.org/articulo. oa? id=47317815006. Fecha de consulta: 24 de mayo de 2016.

12. Cardona A. D, Segura C Á M, Berbesí F D Y, Prevalencia y factores asociados al síndrome de sobre- carga del cuidador primario de ancianos. Revista Facultad Nacional de Salud Pública 20133130-39. Disponible en: http://www.redalyc.org/articulo. oa?id=12026437003. Fecha de consulta: 24 de mayo de 2016.

13. Morales Mora Rafael; Grado de sobrecarga y afectación a la salud, en el cuidador del paciente inmovilizado perteneciente a la unidad de atención comunitaria geriátrica del hospital nacional de geriatría y gerontología; Revista Médica de Costa Rica y Centroamérica LXXII (614) 225 - 230, 2015; Citado: Enero 2016. http://www.binasss.sa.cr/revistas/ rmcc/614/art41.pdf

14. Palome V. Gabriela; Nivel de sobrecarga en el cuidador principal del paciente crónico hospitalizado; Digital ciencia@UAQRO; Querétaro 2014; Citado: Enero 2016. http://www.uaq.mx/investigacion/revista_ciencia@uaq/ArchivosPDF/v7-n1/03Articulo. pdf.

15. García-Calvente María; El impacto de cuidar en la salud y la calidad de vida de las mujeres; Gaceta Sanitaria v.18 supl.2; Barcelona mayo 2014; Citado: Enero 2016. Disponible en: http://scielo.isciii.es/ scielo.php?pid = S021391112004000500011\&script $=$ sci_arttext\&tlng $=$ en

16. Ruiz María; Cansancio, cuidados y repercusiones en cuidadores informales de adultos mayores con enfermedades crónicas degenerativas; Universidad Juárez Autónoma de Tabasco 2015; Citado: Enero 2016. http://formacionasunivep.com/ejihpe/index.php/ journal/article/view/66/53 